\title{
The Activation of Muscle Spindles Enhances the Thixotropic Behavior of Rib Cage Respiratory Muscles
}

\author{
Masahiko SHIBATA, Masahiko IZUMIZAKI, and Ikuo HOMMA \\ Second Department of Physiology, Showa University School of Medicine, Tokyo, 142-8555 Japan
}

\begin{abstract}
Rib cage inspiratory muscle contractions at a deep inflated position caused a subsequent increase in rib cage cross-sectional area at the end-expiratory phase. The increase in this area was enhanced by the activation of muscle
\end{abstract}

spindles in the inspiratory muscles by a mechanical vibration combined with muscle contractions. [The Japanese Journal of Physiology 53: 243$246,2003]$

Key words: chest wall vibration, tonic vibration reflex, functional residual capacity.

Skeletal muscle fibers have complex biophysical properties that make the stiffness and resting tension at a given muscle length dependent on the previous history of movements and contractions [1-4]. These history-dependent properties are referred to as thixotropy. Muscle thixotropy is generally believed to depend on the formations and detachments of cross bridges between sliding actin and myosin filaments [3-6]. Homma and Hagbarth showed that rib cage respiratory muscles have thixotropic properties similar to those observed in other skeletal muscles [7]. In their study, the end-expiratory position (EEP) of the rib cage judged by its cross-sectional area measured with respiratory induction plethysmography is temporarily elevated after deep inspirations combined with forceful voluntary contraction of inspiratory rib cage muscles with airway occlusion. They also showed that the EEP elevation was attenuated without the voluntary contraction. These history-dependent changes in EEP can be explained in terms of muscle thixotropy.

Evidence suggests that voluntary muscle contraction at a muscle length is necessary for the development of muscle thixotropy [3, 8]. Voluntary muscle contraction activates $\gamma$ motoneurons via $\alpha-\gamma$ linkage to maintain continuous feedback from muscle spindles. Afferent information from the spindles induces reflex contraction of the same muscle when the muscle does not shorten during contraction. Thus an increased afferent discharge from muscle spindles in rib cage inspiratory muscles very likely occurs when a forceful inspiratory effort is made with airway occlusion, which may contribute to the thixotropic behavior of rib cage inspiratory muscles.

Mechanical vibration applied to a skeletal muscle induces an involuntary tonic reflex contraction of the muscle [9]. The main afferent source of this tonic vibration reflex (TVR) is generally considered to be the primary muscle spindle endings with their excitatory projections to homonymous $\alpha$ motoneurons [10]. Similar tonic contractions in response to chest wall vibration (CWV) have been shown in human intercostal muscles [11]. The aim of the present study was to investigate whether the activation of muscle spindles in rib cage inspiratory muscles with CWV during forceful inspiratory effort affected the thixotropic behavior of the muscles.

The study was performed on 6 normal subjects (all males aged 21 to 34) with no history of chronic pulmonary or neuromuscular disease. All subjects were naive to the purpose of the study and signed an informed consent, and this study was approved by the Ethical Committee of Showa University. The subjects wore a mouthpiece, which was connected to a spirometer (ML140spilometer, ADInstruments, Castle Hill, Australia) and to a magnetically driven shutter for airway occlusion. A transducer (TP-604T, Nihon Kohden, Tokyo, Japan) attached to the mouthpiece measured the mouth pressure. The cross-sectional area of the rib cage was measured in a supine position with respiratory induction plethysmography by using a

Received on January 20, 2003; accepted on May 3, 2003

Correspondence should be addressed to: Masahiko Izumizaki, Second Department of Physiology, Showa University School of Medicine, Tokyo, 142-8555 Japan. Tel: +81-3-3784-8113, Fax: +81-3-3784-0200, E-mail: masahiko@med.showa-u.ac.jp 
Respitrace transducer (Ambulatory Monitoring, Ardsley, USA) as previously described [7]. The EEP values of the rib cage from each of five breaths before conditioning were averaged, and the averaged value was defined as zero level. Breath-by-breath values between the zero line and the EEP of the rib cage were measured, and all data were fed into an analysis system (PowerLab, ADInstruments).

Homma et al. showed that intercostal muscles in the upper and the lower regions had inspiratory and expiratory functions, respectively [11]. Two cylindertype vibrators (Minato Medical, Osaka, Japan) were attached bilaterally at the second or third interspaces at the parasternal region (upper CWV) to activate muscle spindles in inspiratory muscles or at the seventh to ninth interspaces anterior to the midaxillary line (lower CWV) to activate muscle spindles in expiratory muscles, producing mechanical oscillations with an amplitude of about $1 \mathrm{~mm}$ and a frequency of $100 \mathrm{~Hz}[12,13]$. In each subject, we studied the aftereffects of 3 different types of conditioning maneuvers on EEP of the rib cage cross-sectional area: (1) forceful inspiratory effort after deep inspiratory rib cage movement (FII) maneuver. Quiet breathing was temporarily interrupted by a deep inflation of the rib cage. The airway was closed while the rib cage remained inflated, and the subject was instructed to make a forceful inspiratory effort for 3 to $4 \mathrm{~s}$ at the inflated position, using the rib cage respiratory muscles. After the efforts, the airway was opened, and quiet breathing was resumed. (2) FII with upper CWV maneuver. Upper CWV was applied simultaneously during the forceful inspiratory effort. (3) FII with lower CWV maneuver. Lower CWV was applied during the forceful inspiratory effort.

The rib cage records in Figs. 1-3 show typical examples of respiratory rib cage movements with mouth pressure for five breaths before and after each conditioning maneuver. The EEP values after these 3 types of conditioning maneuvers showed an obvious tendency to elevate (Figs. 1-3). The bar histograms in Fig. 4 illustrate the pooled results of the EEP measurements after the conditioning maneuvers, showing that EEPs after FII with upper CWV were more enhanced than those after the other two maneuvers, FII with lower CWV and FII without CWV. In all conditioning maneuvers, the EEPs reached their peaks at the first breath, and the aftereffects of maneuvers on EEP slowly subsided during the following period. EEP values were analyzed with a two-way repeatedmeasures analysis of variance to test for within-factor (breath course and maneuver type) effects. The breath immediately preceding each maneuver was employed
A

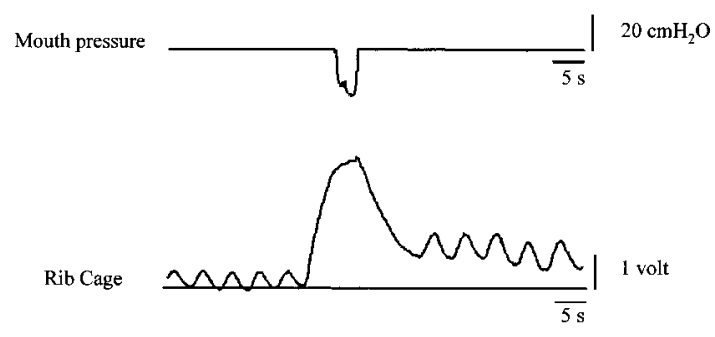

B

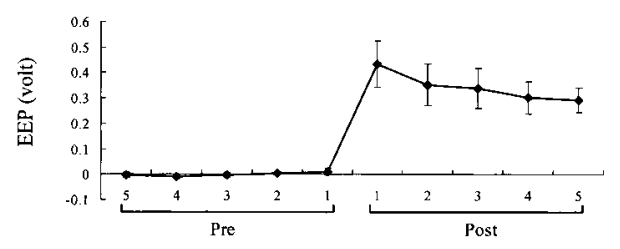

Fig. 1. Forceful inspiratory effort after deep inspiratory rib cage movement (FII). A: A typical example of FII. The top trace represents the mouth pressure, and the middle trace represents the aftereffects of FII on end-expiratory position (EEP) levels with respiratory induction plethysmography, i.e., the chest wall positions at the end of the expiratory phases of a spontaneous breath cycle. Upward deflections in the rib cage trace indicate inspiratory movements; the horizontal line indicates mean EEP level of the 5 breaths taken immediately before FII. B: Pooled results of the aftereffects of FII in all 6 subjects before and after FII. EEP was elevated after FII. It reached a peak at the first breath, and the aftereffects slowly subsided during the following period. Data shown are means \pm SEM values.

A
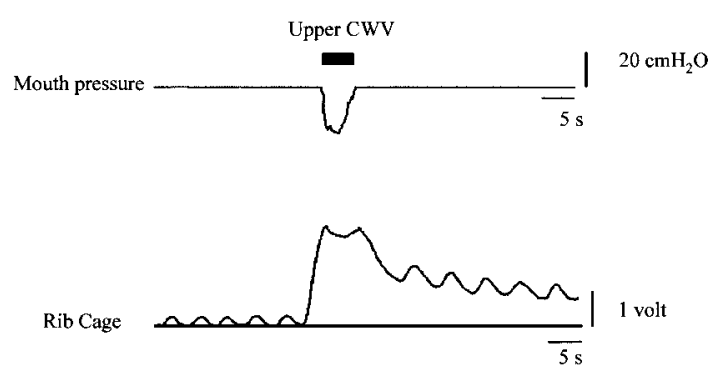

B

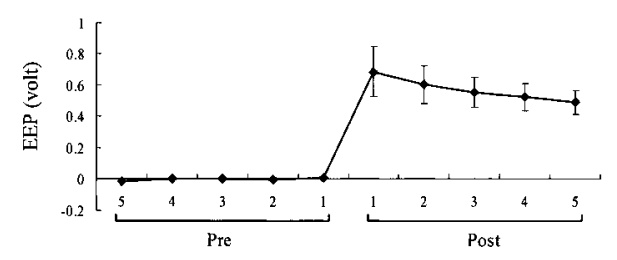

Fig. 2. Fll combined with upper chest wall vibration (CWV). A: An example of the aftereffects of Fll with upper CWV on EEP. B: Pooled results of the aftereffects of FII with upper CWV in all 6 subjects. 
A
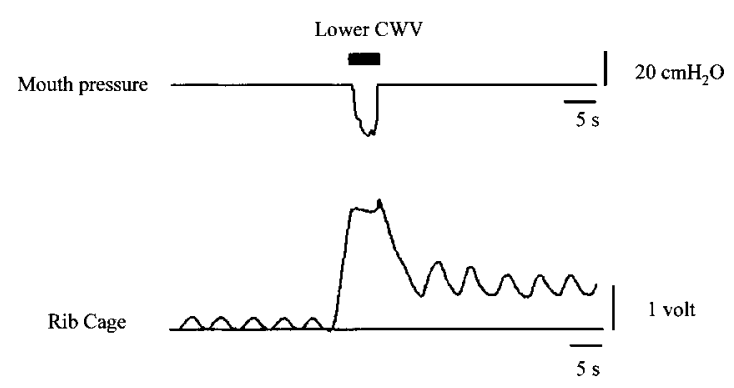

B

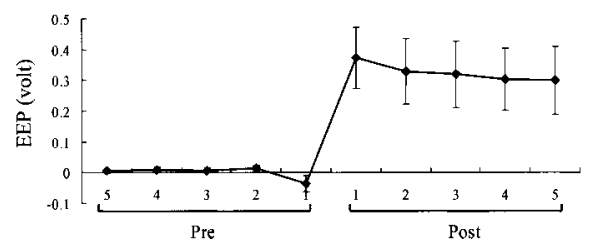

Fig. 3. Fll combined with lower CWV. A: An example of the aftereffects of Fll with lower CWV on EEP. B: Pooled results of the aftereffects of FII with lower CWV in all 6 subjects.

as a control value. There were significant main effects for both breath course and maneuver type (both $p<0.01$ ). Additional comparisons were made at each breath. EEP levels after FII with upper CWV were significantly higher than those after FII without CWV and with lower $\mathrm{CWV}$ from the first to the fourth breath (all $p<0.01$, one-way repeated-measures analysis of variance with a post hoc Bonferroni multiple comparisons test).

We found that rib cage inspiratory muscle contraction at an inflated position caused a subsequent elevation in EEP and that upper CWV combined with rib cage inspiratory muscle contraction resulted in moreelevated EEP, whereas lower CWV provided no additional shifts for EEP.

Upper CWV during quiet breathing produces an upward shift of the rib cage by TVR, and the rib cage position returns to its baseline level immediately after the cessation of upper CWV [11]. However, in the present study, we showed that upper CWV has aftereffects on the rib cage position when combined with muscle thixotropy. Our results suggest that an increase in afferent discharge from muscle spindle endings of inspiratory muscles during contraction enhanced the thixotropic behavior of the muscles. A reflex contraction of inspiratory muscles because of CWV in combination with voluntary contraction may promote the development of thixotropy of the muscles.

The activation of muscle spindles in rib cage expiratory muscles with lower $\mathrm{CWV}$ during inspiratory muscle contraction failed to have an additional after-

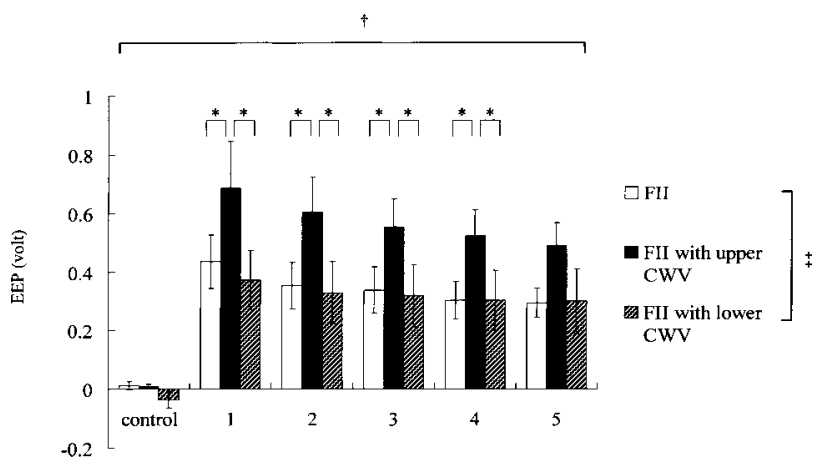

Fig. 4. Aftereffects of FII and FII with upper CWV and of Fll with lower CWV on EEP of post five breaths. The breath immediately preceding each maneuver was employed as a control value. EEP values were analyzed with two-way repeated-measures analysis of variance to test for within-factor (breath course and maneuver type) effects. There were significant main effects for both breath course $\left({ }^{\dagger} p<0.01\right)$ and maneuver type $\left({ }^{\ddagger} p<0.01\right)$. EEP levels after FII with upper CWV were significantly higher than those after FII without CWV and with lower CWV $\left({ }^{*} p<0.01\right)$ from the first to the fourth breaths. No statistical interaction was found. Data shown are means \pm SEM values.

effect on EEP. Muscle thixotropy was not expected to occur in rib cage expiratory muscles because conditioning maneuvers included neither a shortening nor a contraction of expiratory muscles. We believe that the activation of muscle spindles in rib cage respiratory muscles provides an aftereffect for EEP in the presence of thixotropy of the muscles.

Thixotropy of respiratory muscles may have a physiological advantage in our everyday lives. A transient and deep inspiration is sufficient to cause a temporary rise in EEP of succeeding breath cycles in healthy subjects [7]. Increased lung volume has the potential advantage of dilating airways. When ventilatory demand increases with deep breaths, the thixotropy of respiratory muscles may be helpful to reduce work against airway resistance. However, it also has potential disadvantages, especially in patients with chronic obstructive pulmonary disease (COPD). Pulmonary hyperinflation in COPD is characterized by an abnormal increase in FRC resulting from a decrease in the elastic recoil of the lungs [14]. As pulmonary hyperinflation progresses, inspiratory muscle fibers must work against not only the elastic recoil of the lungs, but also that of the rib cage. Thus inspiratory effort requires more negative intrathoracic pressure to expand the lungs with shortened inspiratory muscle fibers, which may be followed by muscle thixotropy.

The present study showed that forceful inspiratory effort at an inflated position caused a subsequent elevation in $\mathrm{EEP}$, and CWV on rib cage inspiratory muscles combined with inspiratory effort caused a more- 


\section{SHIBATA, M. IZUMIZAKI, and I. HOMMA}

elevated EEP. This suggests that the activation of muscle spindles of rib cage respiratory muscles during respiratory effort has an aftereffect on thixotropic behavior of the muscles.

We thank Suzanne Knowlton for preparation of the manuscript.

\section{REFERENCES}

1. Lakie M, Walsh EG, and Wright GW: Resonance at the wrist demonstrated by the use of a torque motor: an instrumental analysis of muscle tone in man. J Physiol (Lond) 353: 265-285, 1984

2. Hagbarth KE, Hagglund JV, Nordin M, and Wallin EU: Thixotropic behaviour of human finger flexor muscles with accompanying changes in spindle and reflex responses to stretch. J Physiol (Lond) 368: 323-342, 1985

3. Proske U, Morgan DL, and Gregory JE: Thixotropy in skeletal muscle and in muscle spindles: a review. Prog Neurobiol 41: 705-721, 1993

4. Gregory JE, Wise AK, Wood SA, Prochazka A, and Proske U: Muscle history, fusimotor activity and the human stretch reflex. J Physiol (Lond) 513: 927-934, 1998

5. Campbell KS and Lakie M: A cross-bridge mechanism can explain the thixotropic short-range elastic component of relaxed frog skeletal muscle. J Physiol (Lond) 510: 941-962, 1998
6. Proske $U$ and Morgan DL: Do cross-bridges contribute to the tension during stretch of passive muscle? J Muscle Res Cell Motil 20: 433-442, 1999

7. Homma I and Hagbarth KE: Thixotropy of rib cage respiratory muscles in normal subjects. J Appl Physiol 89: 1753-1758, 2000

8. Hagbarth KE and Nordin M: Postural after-contractions in man attributed to muscle spindle thixotropy. J Physiol (Lond) 506: 875-883, 1998

9. Hagbarth KE and Eklund G: Motor effects of vibrating stimuli in man. In: Muscular Afferents and Motor Control, ed. Graint R, Almqvist \& Wiskell, Stockholm, pp 177-186, 1966

10. Eklund $G$ and Hagbarth KE: Normal variability of tonic vibration reflexes in man. Exp Neurol 16: 80-92, 1966

11. Homma I, Eklund G, and Hagbarth KE: Respiration in man affected by TVR contractions elicited in inspiratory and expiratory intercostal muslces. Respir Physiol 35: 335-348, 1978

12. Homma I, Obata T, Sibuya M, and Uchida M: Gate mechanism in breathlessness caused by chest wall vibration in humans. J Appl Physiol 56: 8-11, 1984

13. Sibuya M, Yamada M, Kanamaru A, Tanaka K, Suzuki $\mathrm{H}$, Noguchi E, Altose MD, and Homma I: Effect of chest wall vibration on dyspnea in patients with chronic respiratory disease. Am J Respir Crit Care Med 149: 1235-1240, 1994

14. Gibson GJ and Pride NB: Lung distensibility. The static pressure-volume curve of the lungs and its use in clinical assessment. Br J Dis Chest 70: 143-184, 1976 